\title{
Simulation of Electrical Characteristics for Misalignment of Coil in Wireless Power Transfer System Using Repeating Coil
}

\author{
Akihiro Imakiire $^{\mathrm{a}, *}$, Hideaki Tokunaga ${ }^{\mathrm{a}}$, Akihiro Kawagoe ${ }^{\mathrm{a}}$, Masahiro Kozako ${ }^{\mathrm{a}}$, Masayuki Hikita ${ }^{\mathrm{a}}$

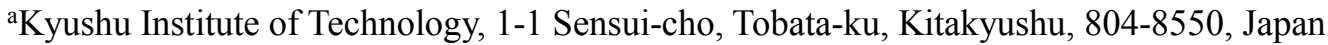 \\ *Corresponding Author: imakiire@ele.kyutech.ac.jp
}

\begin{abstract}
This paper compares the electrical characteristics of the S-P type and the repeating coil type wireless power transfer (WPT) system. The features of the two types of WPT systems are described if the output power is same value using the same coil model. The current and voltage values of resonance capacitor are high, and the output power decrease when misalignment of coil occurs. Therefore, compensation method for misalignment is investigated to suppress this burden of resonance capacitor when misalignment of coil occurs. These results are calculated from circuit equations based on the results of electromagnetic analysis and are investigated for automatic guided vehicle (AGV) applications. Thus, if the repeating coil maintains the center position between the primary and secondary coils, the repeating coil type can suppress the burden of the resonance capacitor and improve the transmission power.
\end{abstract}

Keywords: wireless power transfer, repeating coil, automatic guided vehicle, relay coil.

\section{Introduction}

In recent years, the wireless power transfer (WPT) system has been researched for various applications, such as electric vehicles (EV). There are numerous circuit configurations with different electrical characteristics ${ }^{(1-4)}$. The circuit configuration of the S-P type WPT system is commonly used for EV applications, which has two resonance capacitors: one is connected in series with the primary coil, and the other is connected in parallel with the secondary coil. On the other hand, the WPT system with a repeating coil (repeating coil type) is well known for its robust performance in the coil position. The electrical characteristic against misalignment of the coil is important to transmit power to the load with high efficiency. The electrical characteristics of other coil types along with the S-P type, and the repeating coil type have been compared by many researchers ${ }^{(1,4)}$. However, it is difficult to compare the electrical characteristics in the same condition, because all the types have different features, circuit configurations, and coil shapes. Thus it becomes difficult to determine a condition that is suitable for both types of the WPT system. Moreover, several different parameters and electrical characteristics exist, consuming lot of time to conduct a simulation and investigation.

This paper compares the electrical characteristics of the S-P type and the repeating type WPT system. The features of the two types WPT system are described if the output power is the same value using same coil model. The current and voltage values of the resonance capacitor are high when misalignment of the coli occurs. Therefore, compensation method is investigated to suppress this burden of resonance capacitor when misalignment of coil occurs. These results are calculated from circuit equations based on the results of electromagnetic analysis and are investigated for automatic guided vehicle (AGV) applications. It is useful to search for optimum design conditions in the WPT system.

In literature (5), the burden of the resonance capacitor in the WPT system was investigated. In literatures (6) and (7), compensation method for misalignment using control or coil shapes were studied. In addition, The Literature (8)-(10) showed the two types dynamic wireless power transfer system such as using parallel line feeder or relay coil(repeating coil), respectively. On the other hand, we have been studied the WPT system using the repeating coil which is controlled the position ${ }^{(11-12)}$. In this paper, we investigate the electrical characteristics, compensation method, and the burden of the capacitor, when the repeating 


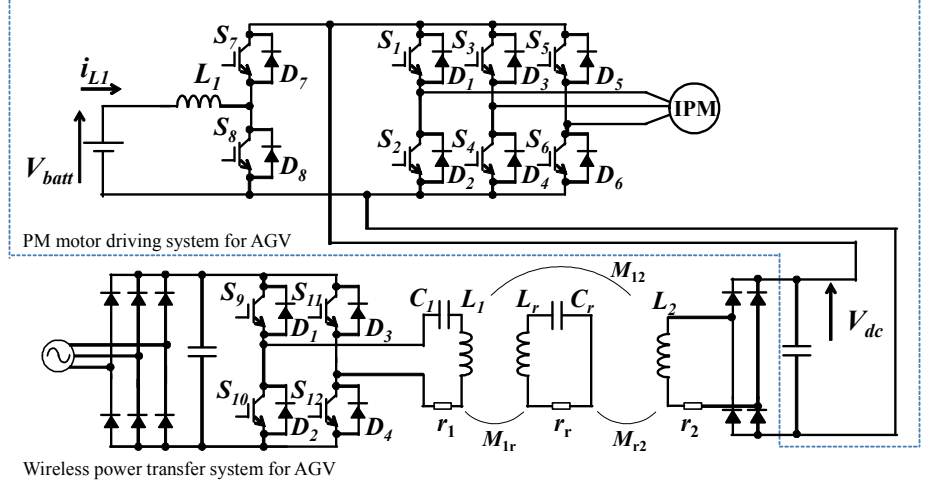

(a) System configuration
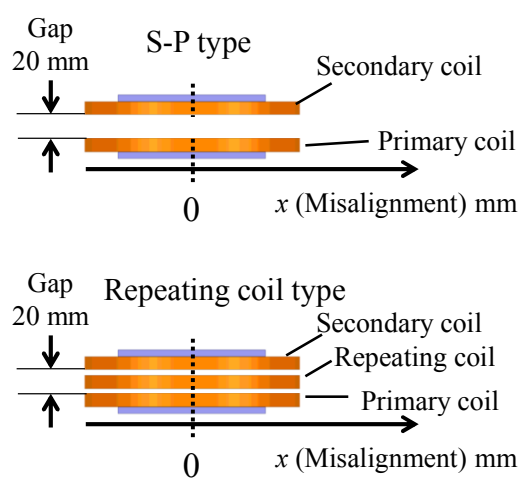

(b) Coils model, and motion of repeating coil and secondary

Fig. 1. Wireless Power Transfer System with Repeating Coil for Automatic Guided Vehicle.

Table 1. Simulation condition.

\begin{tabular}{|c|c|}
\hline Parameters & Value \\
\hline Input voltage $V_{1}$ (S-P type) & $141 V_{\text {rms }}$ \\
\hline Input voltage $V_{1}$ (Repeating coil type) & $141 V_{\text {rms }}$ \\
\hline $\begin{array}{l}\text { Input voltage } V_{1}^{\prime} \text { (Repeating coil type) } \\
\text { The output power is same value in the S-P type } \\
\text { and the repeating coil type }\end{array}$ & $254 V_{\text {rms }}$ \\
\hline Frequency of input voltage $V_{1}$ & $25 \mathrm{kHz}$ \\
\hline Gap length $G$ & $20 \mathrm{~mm}$ \\
\hline Misalignment $X$ & $0-80 \mathrm{~mm}$ \\
\hline Load resistance $R_{\mathrm{L}}$ & $100 \Omega$ \\
\hline Inductance $L_{1}(\mathrm{~S}-\mathrm{P}$ type $) @$ No misalignment & $638 \mu \mathrm{H}$ \\
\hline Inductance $L_{2}(\mathrm{~S}-\mathrm{P}$ type $) @$ No misalignment & $637 \mu \mathrm{H}$ \\
\hline $\begin{array}{c}\text { Inductance } L_{1} \text { (Repeating coil type) } \\
\text { @No misalignment }\end{array}$ & $573 \mu \mathrm{H}$ \\
\hline $\begin{array}{c}\text { Inductance } L_{\mathrm{r}}(\text { Repeating coil type }) \\
@ \text { No misalignment }\end{array}$ & $638 \mu \mathrm{H}$ \\
\hline $\begin{array}{c}\text { Inductance } L_{2} \text { (Repeating coil type) } \\
@ \text { No misalignment }\end{array}$ & $638 \mu \mathrm{H}$ \\
\hline Resonance capacitor $C_{1}(\mathrm{~S}-\mathrm{P}$ type $)$ & $92 \mathrm{nF}$ \\
\hline Resonance capacitor $C_{2}$ (S-P type) & $64 \mathrm{nF}$ \\
\hline Resonance capacitor $C_{1}$ (Repeating coil type) & $71 \mathrm{nF}$ \\
\hline Resonance capacitor $C_{\mathrm{r}}$ (Repeating coil type) & $71 \mathrm{nF}$ \\
\hline Internal resistance of coil $r_{1}, r_{\mathrm{r}}, r_{2}$ & $0.173 \Omega$ \\
\hline Relative permeability of ferrite core & 2300 \\
\hline Ferrite core size & $\begin{array}{c}125 \times 125 \times 5 \\
{[\mathrm{~mm}]}\end{array}$ \\
\hline Diameter of coil & $\Phi 175 \mathrm{~mm}$ \\
\hline Number of turns of coil & 75 \\
\hline
\end{tabular}

coil maintains at the center of the primary and secondary coils.

\section{Wireless power transfer system configuration for automatic guided vehicle}

\subsection{Overall WPT system for AGV}

Figures 1 (a) and (b) show the WPT system for AGV investigated thus far. Figure 1(a), shows the voltage booster that controls the battery current, DC link capacitor used commonly in the WPT system, and three phase inverter for motor of AGV. Figure 1 (b) shows the coil model and motions of repeating coil and secondary coils. The secondary coil position means the AGV position. In contrast, the repeating coil is set at the center between the primary and secondary coils to keep the output voltage constant. In other words, the repeating coil is controlled to suppress the decrease in the output power.

\subsection{Simulation model of the $S-P$ type and the repeating coil type}

Figure 2 shows the simulation model of the S-P type and the repeating coil type. In both these types, the coil shape and the number of turns are similar. However, with addition of the repeating coil type, the repeating coil is set at the center of the primary and secondary coils. The gap length and misalignment is defined in Fig. (1). In the simulation, misalignment is a parameter. The simulation condition, physical property values, and size of coil are summarized in Table 1. In addition, the input voltage $V_{1}$ and the load resistance $R_{\mathrm{L}}$ were set as sine waveforms and $100 \Omega$, respectively, in the simulation.

First, the output voltage and current of coil were simulated using electromagnetic analysis (ANSYS, Maxwell and Simplorer), and the inductance and mutual inductance were calculated as parameters as shown in Fig. 


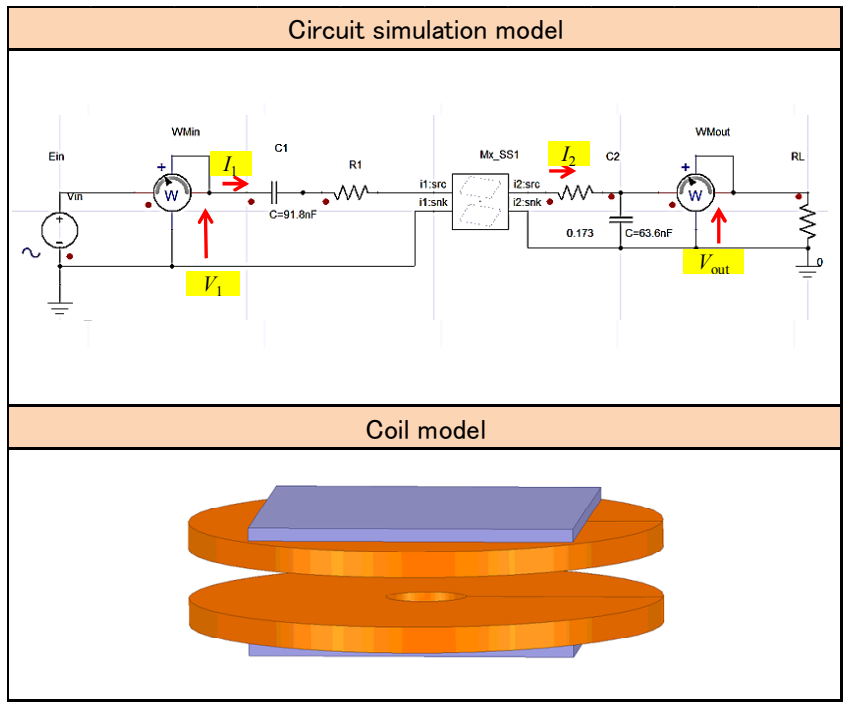

(a) The S-P type

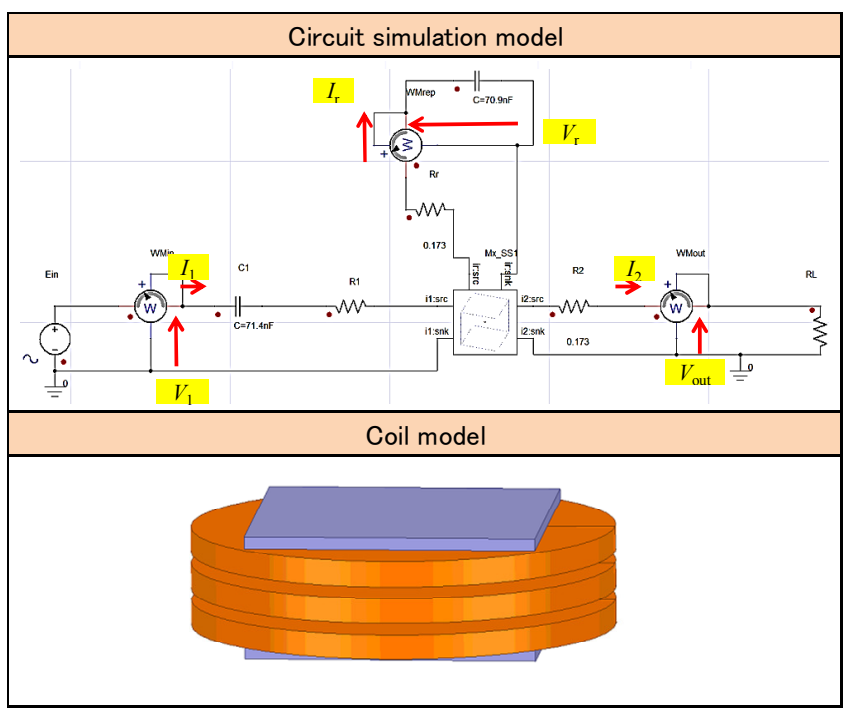

(b) The repeating coil type

Fig. 2 Simulation model using electromagnetic analysis.

2.

Next, the simulation results using electromagnetic software and the calculation results using circuit equations were compared. As a result, the validity of calculation results was confirmed using circuit equations. Note that the calculation results derived using parameters such as inductance and mutual inductance were simulated using electromagnetic software. The circuit equations are useful to design the WPT system when the influence of the parameter on the electrical characteristics of the WPT system is investigated.

Finally, the S-P type and repeating coil type calculation results were compared and discussed. In addition, a compensation method is described.

$$
\begin{aligned}
& {\left[\begin{array}{c}
V_{1} \\
0
\end{array}\right]=\left[\begin{array}{cc}
r_{1}+j\left(\omega L_{1}-\frac{1}{\omega C_{1}}\right) & -j \omega M_{12} \\
j \omega M_{12} & -r_{2}-j\left(\omega L_{2}-\frac{\frac{R_{L}}{\omega C_{2}}}{R_{L}-j \frac{1}{\omega C_{2}}}\right.
\end{array}\right]\left[\begin{array}{l}
I_{1} \\
I_{2}
\end{array}\right]} \\
& {\left[\begin{array}{l}
V_{1} \\
0 \\
0
\end{array}\right]=\left[\begin{array}{ccc}
r_{1}+j\left(\omega L_{1}-\frac{1}{\omega C_{1}}\right) & -j \omega M_{1 r} & -j \omega M_{12} \\
j \omega M_{1 r} & -\left(r_{r}+j\left(\omega L_{r}-\frac{1}{\omega C_{r}}\right)\right) & -j \omega M_{r 2} \\
j \omega M_{12} & -j \omega M_{r 2} & -\left(r_{2}+j\left(\omega L_{2}-\frac{R_{L}}{\omega C_{2} R_{L}-j}\right)\right)
\end{array}\right]\left[\begin{array}{c}
I_{1} \\
I_{r} \\
l_{2}
\end{array}\right]} \\
& \Rightarrow L_{1}-M_{12} \\
& M_{12}-\frac{\omega M_{1 r} M_{r 2}}{\omega L_{r}-\frac{1}{\omega C_{r}}} \Rightarrow \infty \\
& C_{1}=\frac{1}{\omega^{2}\left(L_{1}+L_{2}-2 M_{12}\right)} \underbrace{\operatorname{mm}}_{L_{1}-M_{12} L_{2}-M_{12}} \prod^{R_{t}}
\end{aligned}
$$

Fig. 3 Equivalent circuit model of the repeating coil type.

\subsection{Equation model of the S-P type and the repeating coil type}

Equations of the S-P type and the repeating coil type are expressed as (1) and (2), respectively. The resonance capacitor value is decided to set the power factor of circuit at 1.0. In addition, the equivalent circuit model of repeating type is shown in Fig. 3. It can be seen that the equivalent circuit model of repeating coil type is similar to that of the S-P type system. Furthermore, if the mutual inductances $M_{1 \mathrm{r}}$ and $M_{\mathrm{r} 2}$ have the same value, and the repeating resonance capacitor value is chosen as the resonance frequency with repeating coil $L_{\mathrm{r}}$, the output voltage can be constant. In this condition, resonance capacitor $C_{2}$ is not needed. Therefore, if the repeating coil position controls the center between the primary and secondary coils, the output voltage can be maintained constant with the input voltage value $V_{\text {in. }}$. Therefore, in the simulation of the repeating coil type, the resonance capacitor $C_{2}$ was not used, as reported in previous investigation ${ }^{(12)}$. The system used repeating resonance capacitor and secondary resonance capacitor. However, electrical characteristics of the S-P type and 
repeating coil type have not compared in detail with similar conditions when the repeating coil is controlled at the center of the primary and secondary coils intentionally.

Therefore, in this paper each WPT system is designed with the same output power, using the same coil model. In addition, the burden of each resonance capacitor was investigated. Furthermore, the change in the impedance locus was observed according to the increase in the misalignment.

Thus, the parameters and the electrical characteristics are large. Thus, we used the circuit equations based on the electromagnetic analysis. However, it is necessary to consider that the S-P type has higher voltage than the repeating coil type as follows.

$$
\begin{gathered}
V_{\text {out }}=\frac{L_{2}}{M_{12}} V_{1} \\
V_{\text {out }}=V_{1}
\end{gathered}
$$

The S-P type and repeating coil type output voltages are expressed as (3) and (4), respectively. In the simulation condition, output power value is the same, i.e., the repeating coil type needs higher voltage inverter than the S-P type to provide the same output power.

\section{Simulation results and discussion}

Figures 4 and 5 show the output voltage and coil current characteristics of the S-P type and the repeating coil type from the electromagnetic analysis and equations (1) and (2), respectively. As shown in the figures, the results obtained from the electromagnetic analysis and the equations are almost similar. It was confirmed that the validity of the results of equations from the results of comparison between the simulation (Fig. 2) and equations. Thus, other characteristics such as coil efficiency, output power, output voltage, and coil current can be calculated by the equations. The inductance and mutual inductance were calculated from the electromagnetic analysis.

Figures 6 (a) and (b) show the output power characteristics for misalignment. For this, the load was set at $100 \Omega$. In addition, Figs. 6 (a) and (b) are different in terms of the input voltage $V_{\text {in }}$ of repeating coil type. In Fig. 6 (a), input voltage $V_{\text {in }}$ of the repeating coil type was set at the same value as that of the S-P type. In (b), input voltage $V_{\text {in }}$ of the repeating coil type was set about two times that of the S-P type to adjust the same output power of the S-P type under the no misalignment.

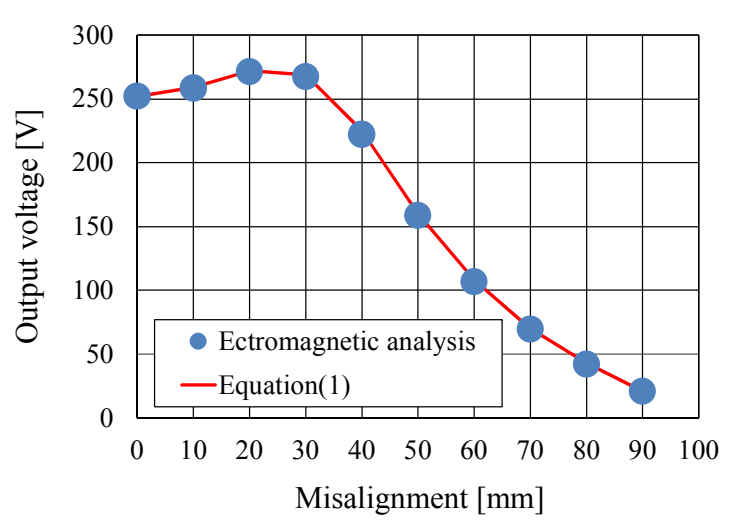

(a) Output voltage

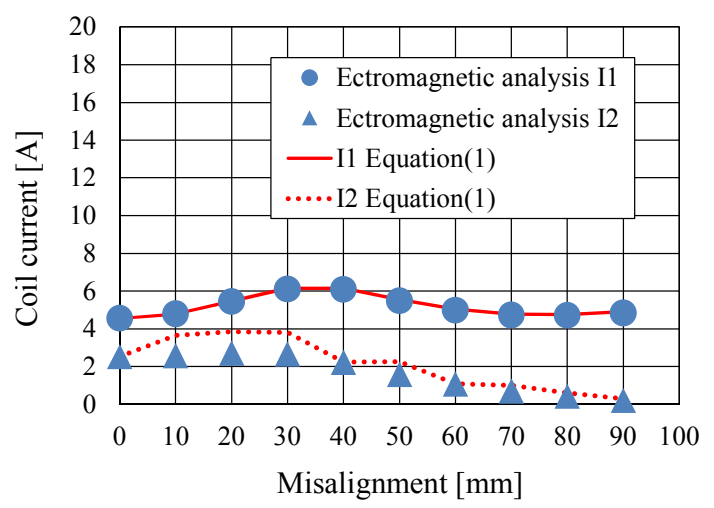

(b) Coil current

Fig. 4 Simulation results of the S-P type.

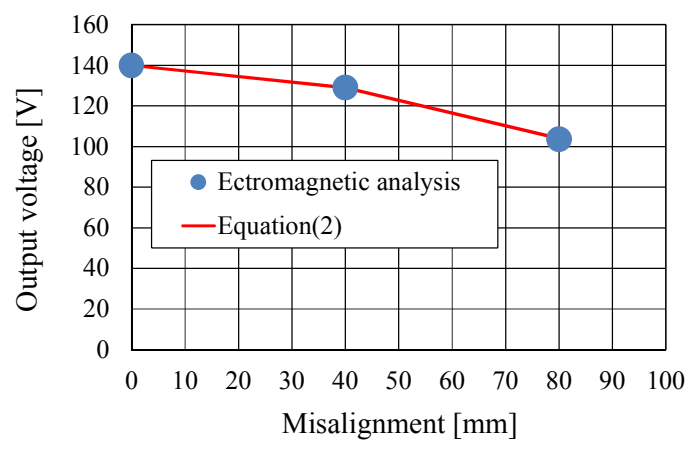

(a) Output voltage

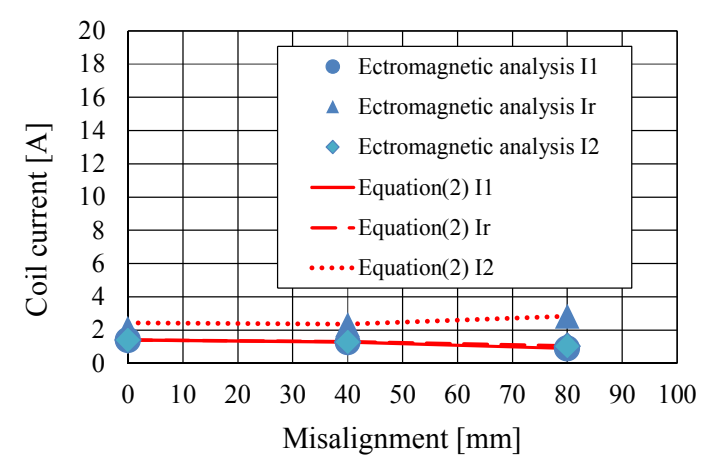

(b) Coil current

Fig. 5 Simulation results of the repeating coil type. 


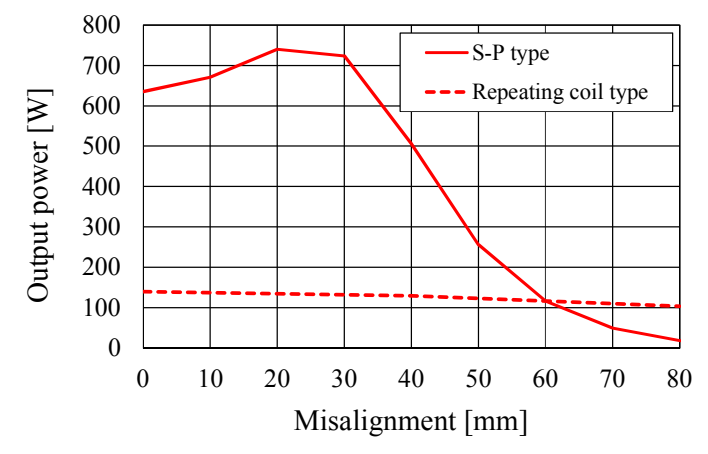

(a) Input voltage value is same the S-P type.

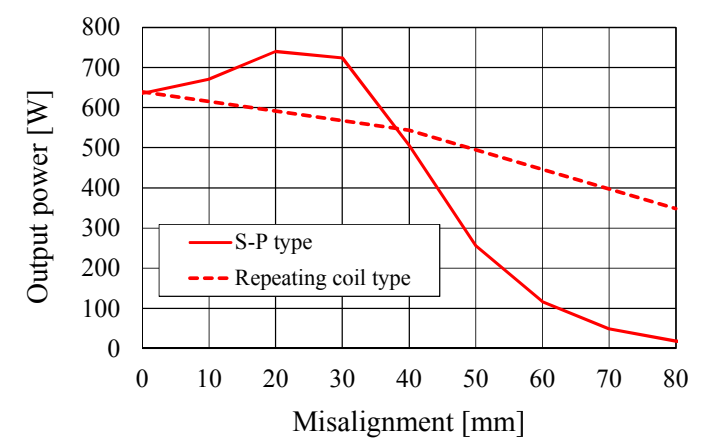

(b) Input voltage value of repeating coil type is two times of that of the S-P type

Fig. 6 Output power for misalignment of two types of WPT system.

From Fig. 6 (a), it was confirmed that the repeating coil type is a more low output voltage and low output power system than the S-P type. These results are expressed by equations (3) and (4). The S-P type is superior to the repeating coil type at the same input voltage $V_{\text {in }}$. However, as shown the Fig. 6 (b), if the output power of the repeating coil type has the same value as the S-P type, then the repeating coil type can be superior to the S-P type in low output power area. For example, observing $400 \mathrm{~W}$, the S-P type can transmit the power about $40 \mathrm{~mm}$ of misalignment. In contrast, the repeating coil type can transmit the power about two times of misalignment than the S-P type.

Next, we compared the coil efficiency of the two types of WPT system. The coil efficiency defined only copper loss of coil.

Figure 7 shows the coil efficiency of the S-P type and the repeating coil type. The repeating coil type has greater efficiency than the S-P type by increasing the misalignment. This is because the S-P type rapidly decreases the output power, thereby increasing the reactive power. On the other hand, the repeating coil type maintains high efficiency even with low output power.

Figures 8 (a) and (b) show the resonance capacitance burden for misalignment depicting the resonance capacitor

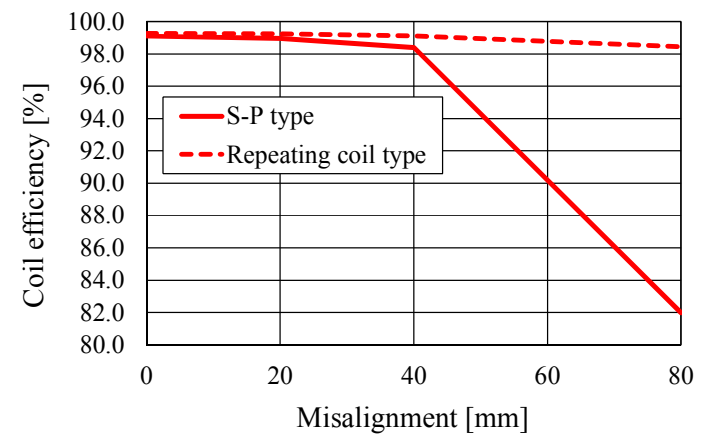

Fig. 7 Coil efficiency for misalignment of the two types of WPT system.

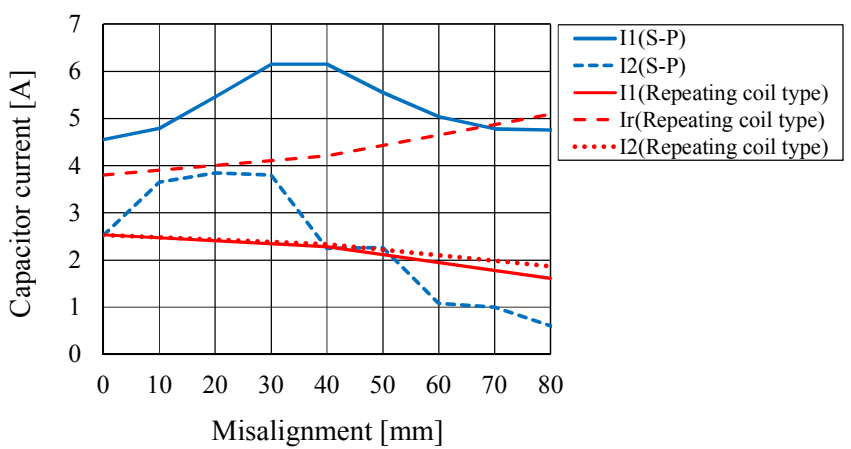

(a) Resonance capacitor current

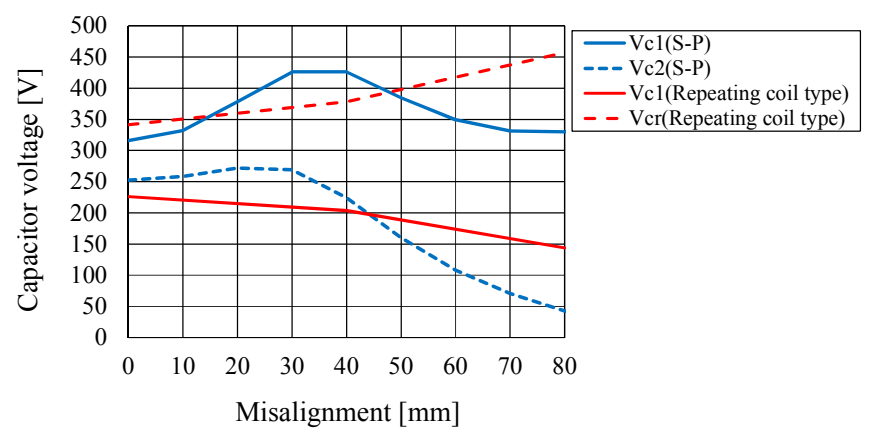

(b) Resonance capacitor voltage

Fig. 8 Resonance capacitor's burden for misalignment of the two types of WPT system.

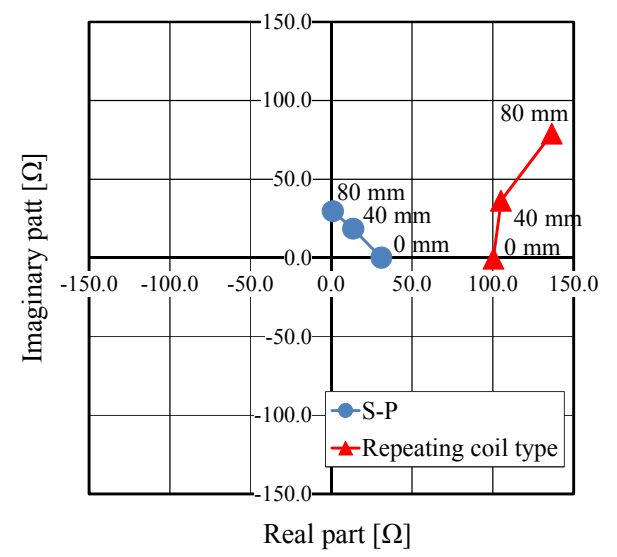

Fig. 9 Impedance locus for misalignment of the two types of WPT system. 
current and voltage characteristics, respectively. The repeating coil type's input voltage $V_{\text {in }}$ was set two times that of the S-P type. Thus, the burden of the primary resonance capacitor increased more than that of the secondary resonance capacitor in the S-P type. In addition, in the repeating coil type, the burden of the repeating resonance capacitor increased more than that of the primary resonance capacitor. Therefore, when designing the repeating coil type, this result of the repeating resonance capacitor's burden should be considered.

Next, we compared the impedance locus for misalignment of the two types WPT system. Figure 9 shows the impedance viewed from the primary side. The circles and triangles indicate the impedances of the S-P type and the repeating coil type, respectively. The result explains the robustness of the repeating coil type. If a misalignment occurs, the leakage inductance increases, and leads to an increase in the reactive power. As shown in Fig. 9, the S-P type shows the almost inductive load at $80 \mathrm{~mm}$ of misalignment. On the other hand, the repeating coil type does not the completely show inductive load at $80 \mathrm{~mm}$ of misalignment. The features of the S-P type and the repeating coil type differ for the impedance locus.

From the equivalent circuit of the repeating coil type as shown in Fig. 3 , if the mutual inductances $M_{1 \mathrm{r}}$ and $M_{\mathrm{r} 2}$ are of the same value and the repeating resonance capacitor's value is chosen as the resonance frequency with repeating coil $L_{\mathrm{r}}$, then the output voltage can be constant. However, the output power (output voltage) gently decreases for misalignment, as shown in Fig. 6.

Figure 10 shows the ideal resonance capacitor value for misalignment of the repeating coil type. As a result, it was confirmed that the change of in ideal $C_{1}$ and $C_{\mathrm{r}}$ values decrease, so the output voltage decrease because it deviates from the designed value. Therefore, additional control is needed to maintain the output voltage.

\section{Compensation method of misalignment and burden for resonance capacitor}

In this section, compensation method of misalignment and burden for resonance capacitor is investigated. In the above simulation, $C_{2}$ was not used in the repeating coil type because $C_{2}$ does not need to adjust the power factor set at 1.0 in no misalignment condition.

Figure 11 shows the output voltage for misalignment of repeating coil type when the secondary resonance capacitor $C_{2}$ is added. The $C_{2}$ was set at the value of $10 \% C_{\mathrm{r}}$ and

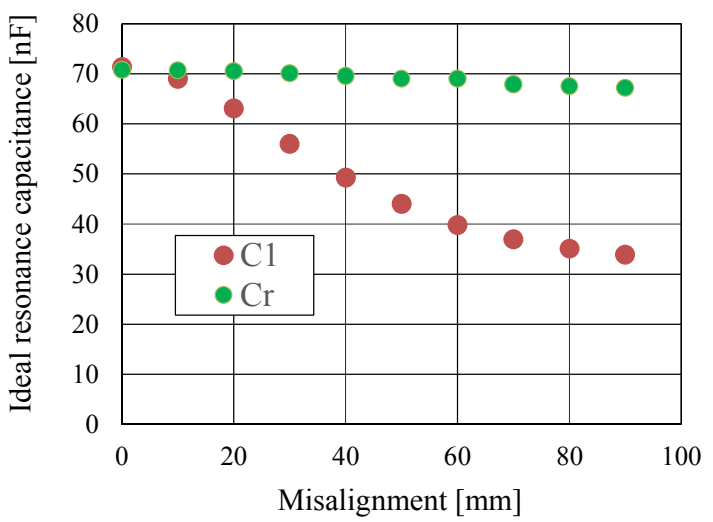

Fig. 10 Ideal resonance capacitor value for misalignment of repeating coil type.

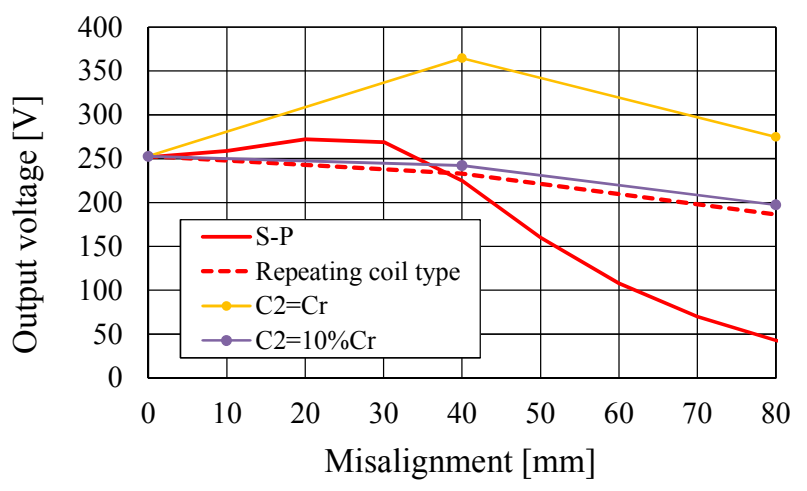

Fig. 11 Output voltage for misalignment of the two types of WPT system when the resonance capacitor $C_{2}$ is added.

$100 \% C_{\mathrm{r}}$. As a result, the output voltage was increased according to the increase in the value of $C_{2}$. Thus, the output power increased for misalignment. The output voltage characteristic of the repeating coil type resembles the S-P type output voltage characteristic with the increase in the value of $C_{2}$. However, the advantage of flat characteristic of the output voltage for misalignment in the repeating coil type is lost on adding $C_{2}$. In addition, the burden of the $C_{1}, C_{\mathrm{r}}$, and $C_{2}$, such as current and voltage characteristics, must be considered for misalignment.

Next, compensation method of misalignment is investigated by controlling the magnitude of input voltage $V_{\text {in }}$ and the frequency of input voltage. As shown in Fig. 9, the impedances from the viewpoint of primary side were resistance and inductive load for misalignment.

Therefore, if the frequency of input voltage $V_{\text {in }}$ is controlled to decrease, then the impedance will change only resistance, and the power factor of the circuit will be controlled at 1.0. However, the control causes an increase in 
the burden of resonance capacitors $C_{1}$ and $C_{\mathrm{r}}$. In particular, the current and voltage of $C_{1}$ directly increases more than that of $C_{\mathrm{r}}$ because, the impedance from the viewpoint of primary side changed to low value by controlling the frequency.

Figures 12 and 13 show the primary current characteristic for the frequency and impedance locus for misalignment of repeating coil type when the frequency changed, respectively. As a result, it can be seen that the impedance from the viewpoint of primary side changed only the resistance by controlling the frequency. In addition, it was confirmed that primary current increased with decreasing frequency.

Figure 14 shows the input and output voltages for misalignment of repeating coil type when the frequency and input voltage was changed. The input voltage was controlled to keep the output power constant. Although two control parameters exist, such as magnitude of input voltage and the frequency, the output power can be controlled to remain constant.

Figure 15 shows the resonance capacitor voltage for misalignment of repeating coil type when the frequency and input voltage was changed. As a, results, the voltage and current of $C_{1}$ over that of $C_{\mathrm{r}}$. The voltage of $C_{1}$ reached 80 $\mathrm{mm}$ of misalignment, which was about four times of $0 \mathrm{~mm}$ of misalignment. The burden of $C_{\mathrm{r}}$ characteristics is similar to that of $C_{1}$.

Finally, the advantages of the repeating coil type is described reflecting the simulation results. By controlling the position of the repeating coil, the output voltage can be kept almost constant. Even if misalignment occurs, the output voltage changes in a safe direction. In addition, the burden of the resonance capacitor for repeating coil is always maximized, thus, the system design is simple.

In particular, an advantage is that the efficiency of the repeating coil type can be kept high even when misalignment occurs. This is also the case when the output power decreases. Therefore, even if the power factor 1 control is not performed, there is no problem if the allowable position range is considered, and the output voltage is designed to achieve the required voltage at that point.

If the power factor 1 control is used, the burden of the primary side resonance capacitor is maximized, and it becomes several times the voltage of the resonance capacitor and the current at the condition of no misalignment. Therefore, when designing the WPT system of repeating coil, the above limitations must be considered.

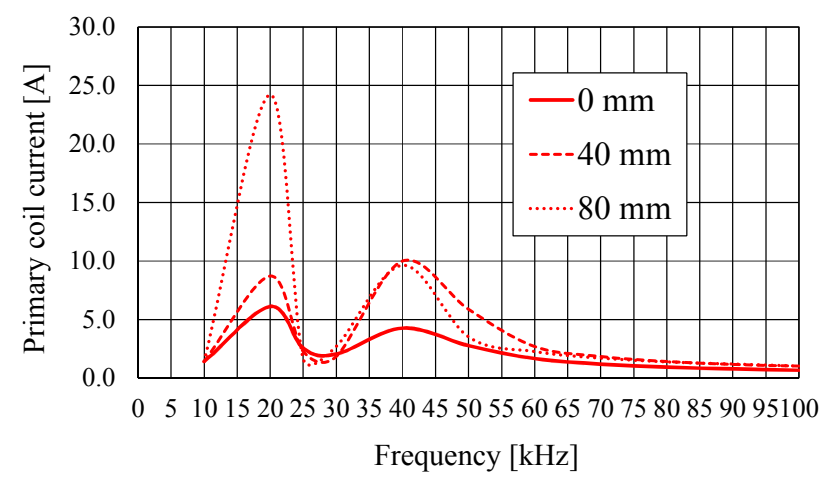

Fig. 12 Primary coil current for misalignment of the repeating coil type.

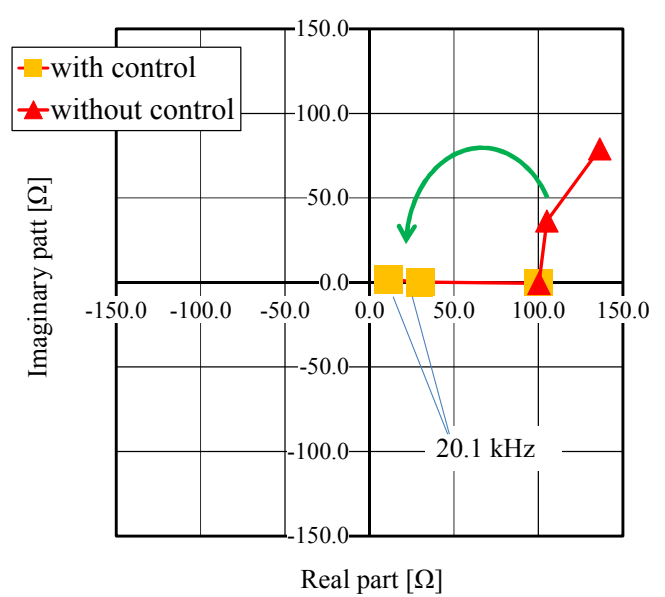

Fig. 13 Impedance locus for misalignment of the repeating coil type when the input voltage and frequency were adjusted.

\section{Conclusions}

This paper compares the electrical characteristics of both the S-P type and the repeating coil type WPT system. The features of the two types was described if the output power is the same using the same coil model when the repeating coil was controlled center of the primary and secondary coils intentionally.

In particular, an advantage is that the efficiency of the repeating coil type can be kept high even when misalignment occurs. This is also the case when the output power decreases. Therefore, even if the power factor 1 control is not performed, there is no problem if the allowable position range is considered, and the output voltage is designed to achieve the required voltage at that point. In addition, the repeating coil type features, such as resonance capacitor burden and impedance locus characteristics, are indicated to design the WPT system. 


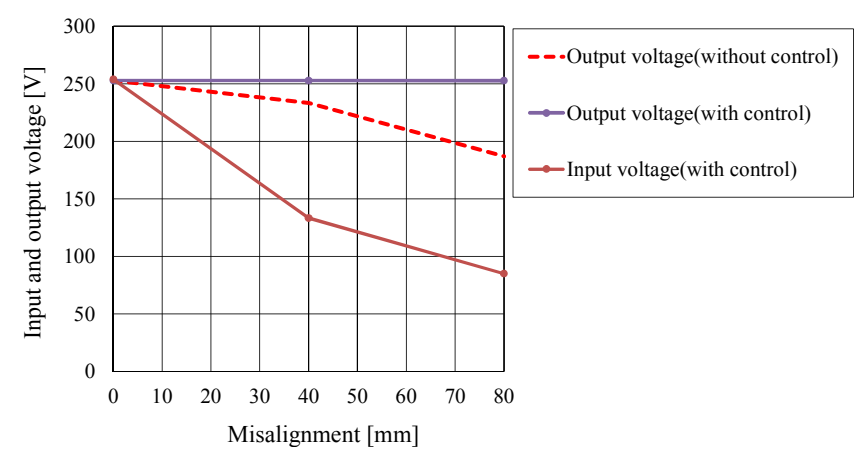

Fig. 14 Input and output voltage for misalignment of the repeating coil type when the input voltage and frequency were adjusted.

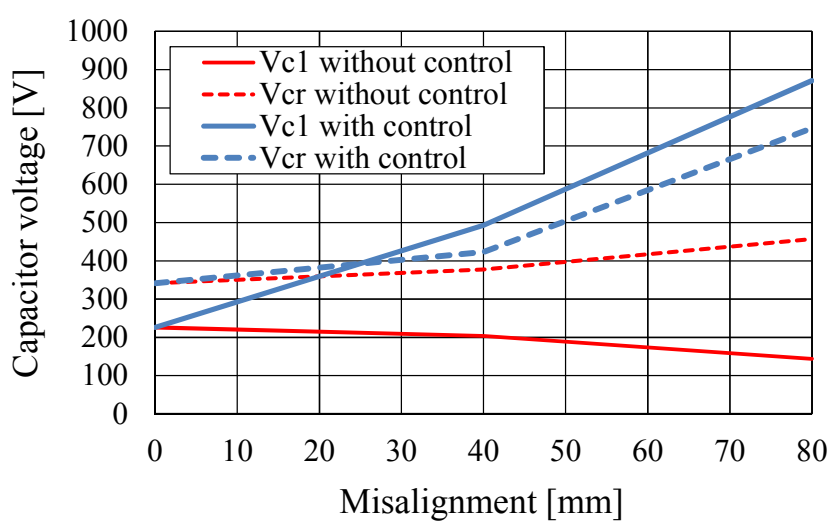

Fig. 15 Resonance capacitor voltage for misalignment of the repeating coil type when input voltage and frequency were adjusted.

\section{Acknowledgment}

This work was supported by JSPS KAKENHI Grant Number $15 \mathrm{~K} 18022$. We would like to acknowledge the concerned members.

\section{References}

(1) T. Kudo, T. Toi, Y. Kaneko, S. Abe "Contactless Power Transfer System Suitable for low Voltage and Large Current Charging for EDLCs", Power Electronics Conference (IPEC-Hiroshima 2014 - ECCE-ASIA), pp. 1109-1114 (2014)

(2) X. Qu, H. Han, S. Wong, C. Tse, W. Chen "Hybrid IPT Topologies With Constant Current or Constant Voltage Output for Battery Charging Applications", IEEE Transactions on Power Electronics, pp. 6329-6337 (2015)

(3) K. Inoue, K. Kusaka, J. Itoh "Development of Inductive Power Transfer System for Excavator
Considered Load Disturbance”, 2017 IEE-Japan Industry Applications Society Conference, pp. I-435-I-438 (2017)

(4) T. Imura, Y. Hori "Unified Theory of Electromagnetic Induction and Magnetic Resonant Coupling", IEEJ Transactions on Industry Applications, Vol. 135, No. 6, pp. 697-710 (2015)

(5) K. Yamamoto, T. Maruyama, K. Kondo, T. Kashiwagi "A Method for Designing a High-Power Contactless Power Transformer Considering Reactive Power", IEEJ Transactions on Industry Applications, Vol. 133, No. 3, pp. 378-385 (2013)

(6) S. Kitazawa, K. Kondo, T. Kashiwagi "An Evaluation of Power Flow Control of the Power Conversion Circuit for Contactless Power Transformer Systems at the Coil Misalignment", IEEJ Transactions on Industry Applications, Vol. 133, No. 5, pp. 518-525 (2013)

(7) A. Zaheer, G. Covic, D. Kacprzak "A Bipolar Pad in a 10-kHz 300-W Distributed IPT System for AGV Applications", IEEE Transactions on Industrial Electronics, Vol. 61, pp. 3288-3301 (2014)

(8) T. Higashino, Z. Ma, M. Okada, Y. Tatsuta, Y. Goto, Y. Tsuruda, R. Tanaka "Proposal of Wireless Power Transfer using Parallel Line Feeder", TECHNICAL REPORT OF IEICE., pp. 20-25 (2013)

(9) H. Abe, T. Akiyama, M. Ozaki, H. Kohara "Simple Equivalent Circuit for a Wireless Power Transfer System Using a Repeating Coil and Effects Confirming the Simplification in the Output Voltage Estimation", IEEJ Transactions on Industry Applications, Vol. 135, No. 6, pp. 679-688 (2015)

(10) M. Kato, T. Imura, Y. Hori "Study about Relation of Efficiency between Receiving Antenna Position and Efficiency for Wireless Power Transfer for Moving Vehicle with Relay Antenna", 2012 IEE-Japan Industry Applications Society Conference, pp. 219-222 (2012)

(11) A. Imakiire, M. Hikita, K. Yamamoto "Proposal of PM motor driving system consist of PWM inverter with voltage booster for applying to automated guided vehicle", International Conference on Electrical Machines and Systems (ICEMS), pp. 2052-2057 (2015)

(12) H. Tokunaga, A. Imakiire, M. Kozako, M. Hikita "Clarification of Resonance Method for Wireless Power Transfer System Using Repeating Coil”, 2017 IEE-Japan Industry Applications Society Conference, pp. 453-454 (2017) 